\title{
Difficulty in Swallowing, with Aspiration Pneumonia in Infancy
}

\author{
N. MATSANIOTIS, J. KARPOUZAS, and M. GREGORIOU \\ From the Paediatric Clinic of Athens University, Athens, Greece
}

Difficulty in swallowing, starting soon after birth and resulting in aspiration pneumonia, may be due to: pharyngeal incoordination; cerebral palsy; familial dysautonomia; poliomyelitis with bulbar palsy; agenesis of bulbar nuclei; or myasthenia gravis.

Pharyngeal incoordination mainly affects premature babies and usually lasts from two to four weeks (Avery, 1964; Scott-Brown, Ballantyne, and Groves, 1965), though a case with pharyngeal incoordination, proved by cineradiography, which lasted much longer, was reported by Benson (1962).

Cerebral palsy may cause difficulty in swallowing, especially during the first year of life. The diagnosis is suggested by a stormy perinatal history, and is later confirmed by the characteristic clinical picture of cerebral palsy, often with athetosis (Woods, 1957).

Familial dysautonomia is a rare syndrome characterized by areas of paraesthesia, the absence of lacrimation, mental subnormality, and muscular incoordination. Agenesis of the fungiform and vallate papillae of the tongue is a consistent and pathognomonic finding (Riley, 1957; Smith, Farbman, and Dancis, 1965).

Poliomyelitis during the newborn period, and agenesis of the bulbar nuclei affecting the mechanism of swallowing, are very rare (Brain, 1962; Ford, 1960; Nelson, 1964).

Newborn infants of myasthenic mothers may present with difficulty in swallowing, but this is usually transient and responds well to cholinergic drugs (Macrae, 1954).

The view that difficulty in swallowing may be due to hypotonia of the pharyngeal musculature has also been expressed (P. Sandifer, 1963, personal communication).

\section{Material}

In the past three years 27 full-term infants have been admitted to the Paediatric Clinic of Athens University,

Recieved September 23, 1966. because of difficulty in swallowing and recurrent bronchopneumonia. Of these, 18 proved to have cerebral palsy with athetosis; 3 were thought to have transient pharyngeal incoordination, the condition clearing up within two weeks. In 1 , the diagnosis of familial dysautonomia was made. In the remaining 5, difficulty in swallowing was eventually attributed to hypotonia of the pharyngeal muscles. Clinical details of these 5 cases are set out in the Table, and show that besides difficulty in swallowing, choking spells, vomiting, and recurrent bronchopneumonia were the common features.

Generalized muscular hypotonia was obvious in 3 patients. Tendon reflexes were normal in 2 and weak in 3. The gag reflex was normal in 3 and weak in 2. One infant had arthrogryposis multiplex.

Three infants became free of symptoms at 12,5 , and 8 months, respectively; 1 died at 2 months, and 1 , who is 4 months old, is still having difficulty in swallowing.

All patients were $x$-rayed, both in order to exclude the presence of anatomical defects, and to demonstrate the diversion of the opaque substance into the trachea. Oesophageal reflux was assessed in the Trendelenburg position.

The following case is typical.

\section{Case Report}

A 7-month-old boy was admitted to the hospital because of choking spells, vomiting, and frequent episodes of bronchopneumonia, since birth. He had been fully investigated in other hospitals, and no cause for his difficulties could be found. He had also been put on steroids for a month without any effect.

His mother reported that 'she was very big' during pregnancy because of hydramnios, and that the foetal movements were weak. An older sib of the patient had developed cerebral palsy with athetosis attributed to anoxia during birth.

Examination revealed a normal well-developed infant. Adventitious sounds could be heard over the chest during feeding. An $x$-ray swallow showed deviation of the opaque substance into the trachea (Fig.). In the Trendelenburg position, an obtuse cardio-oesophageal angle and oesophageal reflux were noted.

The patient was put on continuous antibiotic treatment, and given frequent small and semi-liquid feeds.

He became free of symptoms at the age of 12 months. 


\section{Discussion}

In all 5 infants no obvious cause for their difficulty in swallowing was found. However, there are some indications that hypotonia of the pharyngeal musculature could be responsible.

The mother's girth during pregnancy was unduly large in all cases, and excessive liquor was noted at rupture of the membranes. Since the infants had none of the defects that are associated with hydramnios (DeYoung, 1958; Lloyd and Clatworthy, 1958), the accumulation of excessive amniotic fluid in these cases may be attributable to poor swallowing movements of the foetus. The mothers of two infants recalled that foetal movements were weak compared to previous pregnancies.

It is well known that infants with benign congenital hypotonia (Oppenheim) sometimes have difficulty in swallowing, as part of the generalized hypotonia.

Although pharyngeal incoordination cannot be excluded with certainty in these cases, the presence of hydramnios and weak foetal movements, the absence of neurological signs, the presence of the gag reflex, the long duration of symptoms, obvious generalized muscular hypotonia in 3 cases, and arthrogryposis in one, all point to pharyngeal hypotonia rather than to incoordination of the pharyngeal musculature.

Management. The management of such cases is a constant nursing problem. These infants should be protected from food aspiration. Gavage is indicated, but the persistence of symptoms for months makes this difficult.

In our 5 cases, thick feedings were given at the beginning through a tube and soon attempts were made to give them thick, small, and frequent feedings by bottle or spoon. All infants were under continuous antibiotic protection.

Gastrostomy may be considered if the episodes of bronchopneumonia are very frequent and severe.

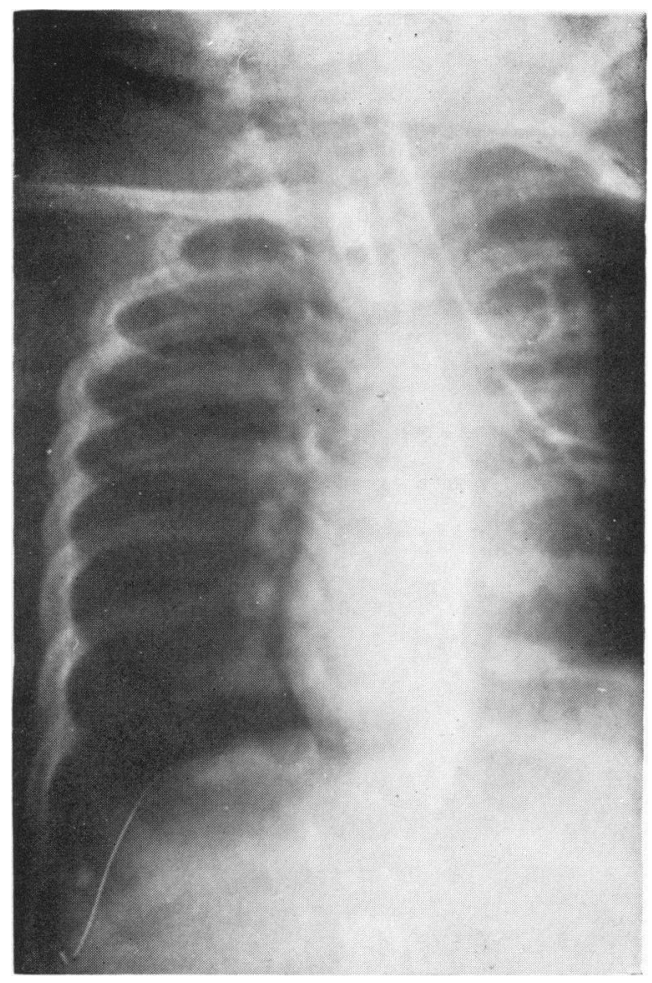

Fig.-Diversion of opaque medium into trachea.

\section{Summary}

Of 27 infants with difficulty in swallowing resulting in aspiration pneumonia, 18 were suffering from cerebral palsy with athetosis, 3 were thought to have pharyngeal muscle incoordination, and 1 had familial dysautonomia.

Five infants are described in whom the aetiology of difficulty in swallowing could not be attributed to any of the known causes.

Generalized muscular hypotonia was present in 3

TABLE

Details of 5 Cases With Difficulty in Swallowing

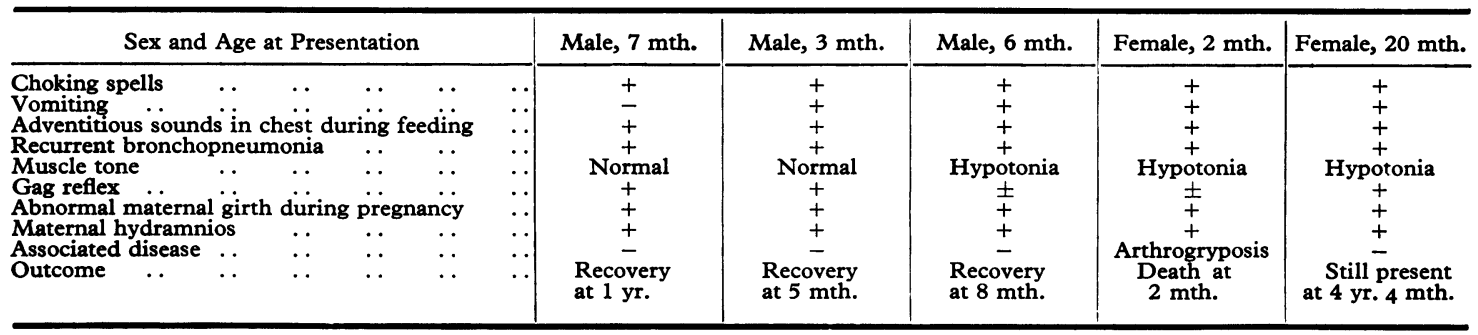


patients. The mothers of all 5 had hydramnios, and 2 reported weak foetal movements.

The hypothesis that the difficulty in swallowing was due to hypotonia of the pharyngeal muscle is discussed.

\section{REFERENCES}

Avery, M. E. (1964). The Lung and its Disorders in the Newborn Infant. W. B. Saunders, Philadelphia.

Benson, P. F. (1962). Transient dysphagia due to muscular incoordination. Proc. roy. Soc. Med., 55, 237.

Brain, W. R. (1962). Diseases of the Nervous System, 6th ed. Oxford University Press, London.

DeYoung, V. R. (1958). Hydramnios as a signal to the physician responsible for newborn infants. $\mathcal{F}$. Pediat., 53, 277.
Ford, F. R. (1960). Diseases of the Nervous System in Infancy, Childhood and Adolescence, 4th ed. Thomas, Springfield, Illinois.

Lloyd, J. R., and Clatworthy, H. W. (1958). Hydramnios as an aid to the early diagnosis of congenital obstruction of the alimentary tract: a study of the maternal and fetal factors. Pediatrics, 21, 903.

Macrae, D. (1954). Myasthenia gravis in early childhood. Pediatrics, 13, 511.

Nelson, W. E. (1964). Textbook of Pediatrics, 8th ed. Saunders, Philadelphia.

Riley, C. M. (1957). Familial dysautonomia. Advanc. Pediat., 9, 186.

Scott-Brown, W. G., Ballantyne, J., and Groves, J. (1965). Diseases of the Ear, Nose and Throat, 2nd ed., Vol. 2. Butterworths, London.

Smith, A. A., Farbman, A., and Dancis, J. (1965). Tongue in familial dysautonomia. Amer. F. Dis. Child. 110, 152.

Woods, G. E. (1957). Cerebral Palsy in Childhood. Wright, Bristol. 